\title{
Tumor neuroendócrino de apêndice: Um raro achado histopatológico incidental após apendicectomia por apendicite perfurada
}

\author{
Appendix neuroendocrine tumor: A rare incidental histopathological findling after perforated \\ appendicitis procedure \\ Apéndice tumor neuroendocrino: Un resultado histopatológico incidental raro después de una \\ apendicitis perforada
}

Diego Leonardo Menezes Maia ORCID: https://orcid.org/0000-0002-5679-9566 Hospital Geral Dr. WaldemarA Alcântara, Brasil E-mail: diegolmaia@ hotmail.com

Kristopherson Lustosa Augusto ORCID: https://orcid.org/0000-0001-9254-9129 Centro Universitário Christus, Brasil Universidade de Fortaleza, Brasil Universidade Federal do Ceará, Brasil E-mail: kristopherson@hotmail.com

Fabrício André Martins da Costa ORCID: https://orcid.org/0000-0002-7080-1386 Hospital Geral Dr. Waldemar Alcântara, Brasil E-mail: fabricioamc18@gmail.com

Gunter Gerson

ORCID: https://orcid.org/0000-0001-9054-253X Laboratório Argos, Brasil

E-mail: gunter_gerson@yahoo.com.br

Suzana Tomaz Vasconcelos ORCID: https://orcid.org/0000-0002-0671-0287 Universidade de Fortaleza, Brasil E-mail: suzy_tomaz_@hotmail.com Alberto Jorge Castelo Branco Roque ORCID: https://orcid.org/0000-0001-6631-3868 Centro Universitário Christus, Brasil E-mail: alberto.castelo@hotmail.com

\begin{abstract}
Resumo
Introdução: As Neoplasias de apêndice são raras e identificadas em cerca de $1 \%$ de todas as apendicectomias, sendo as etiologias mais comuns em primeiro de origem epitelial e em segundo, os neuroendócrinos. Os tumores neuroendócrinos são derivados das células enterocromafins, produzindo os grânulos Cromogranina A e Sinaptofisina e são o segundo tipo histológico mais comum de neoplasia em apêndice, muitas vezes, encontrados incidentalmente após apendicectomia devido sua apresentação indolente, mas, por vezes, são os causadores da apendicite aguda. Objetivo: Relatar a experiência sobre o caso de um paciente diagnosticado com tumor neuroendócrino de apêndice após apendicite aguda perfurada que havia apresentado, anteriormente, tromboses de vasos abdominais. Relato de caso: Paciente do sexo masculino, 28 anos, há 2 meses com dor abdominal em mesogástrio e epigástrio, sendo diagnosticado inicialmente com trombose intra-abdominal. Neste internamento, apresentou dor abdominal difusa mais proeminente em fossa ilíaca direita com descompressão dolorosa, submetido à videolaparoscopia exploradora e evidenciado apendicite grau 4. À análise histopatológica evidenciado tumor neuroendócrino em apêndice bem diferenciado G1 com tamanho de $2 \mathrm{~mm}$ em maior eixo, índice mitótico com menos de 2 mitoses, estadiamento patológico pT1 pNx, margens livres, invasão linfovascular e perineural. Imuno-histoquímico com índice de proliferação celular (Ki-67) de 1\% e identificação dos componentes Cromogranina A clone policlonal e Sinaptofisina clone DARKSYNAP positivo. Conclusão: Relatamos um caso de um paciente com Tumor Neuroendócrino de apêndice com apresentação inicial como apendicite aguda e, à patologia, limitada ao apêndice sem invasão linfovascular e teve a apendicectomia total como procedimento curativo.
\end{abstract}

Palavras-chave: Apendicectomia; Apendicite; Células neuroendócrinas; Neoplasias; Proliferação de células. 


\begin{abstract}
Introduction: Appendix neoplasms are rare and identified in about $1 \%$ of all appendectomies, with the most common etiologies being first of epithelial origin and second, neuroendocrine. Neuroendocrine tumors are derived from enterochromaffin cells, producing the granules Chromogranin A and Synaptophysin and are the second most common histological type of appendix neoplasm, often found incidentally after appendectomy due to their indolent presentation, but are sometimes the causes of acute appendicitis. Objective: To report the experience of a patient diagnosed with neuroendocrine tumor of the appendix after acute perforated appendicitis who had previously presented abdominal vessel thrombosis. Case report: Male patient, 28 years old, with abdominal pain in mesogastrium and epigastrium for 2 months, being initially diagnosed with intra-abdominal thrombosis. Hospitalized presenting a diffuse abdominal pain, more prominent in the right iliac fossa with painful decompression, submitted to exploratory laparoscopy and evidenced grade 4 appendicitis. Histopathological analysis revealed a differentiated neuroendocrine tumor in appendage G1 with a size of $2 \mathrm{~mm}$, mitotic index with less than 2 mitoses, pT1 pNx pathological staging, free margins, lymphovascular and perineural invasion. Immunohistochemistry with cell proliferation index (Ki-67) of $1 \%$ and identification of the components Chromogranin A polyclonal clone and Synaptophysin clone DARKSYNAP positive. Conclusion: We report a case of a patient with Neuroendocrine Tumor of the appendix with initial presentation as acute appendicitis and, to the pathology, limited to the appendix without lymphovascular invasion, with total appendectomy as a curative procedure.
\end{abstract}

Keywords: Appendectomy; Appendicitis; Neuroendocrine cells; Neoplasms; Cell proliferation.

\title{
Resumen
}

Las neoplasias del apéndice son raras y se identifican en aproximadamente el 1\% de todas las apendicectomías, siendo las etiologías más comunes la primera de origen epitelial y la segunda, la neuroendocrina. Los tumores neuroendocrinos se derivan de células enterocromafines, que producen gránulos de cromogranina A y sinaptofisina y son el segundo tipo histológico más común de neoplasia de apéndice, que a menudo se encuentra de manera incidental después de la apendicectomía debido a su presentación indolente, pero a veces son la causa de apendicitis aguda. Objetivo: Informar la experiencia de un paciente diagnosticado de tumor neuroendocrino de apéndice tras apendicitis aguda perforada que previamente había presentado trombosis de vasos abdominales. Caso clínico: Paciente de sexo masculino, 28 años, de 2 meses con dolor abdominal en mesogastrio y epigastrio, siendo diagnosticado inicialmente de trombosis intraabdominal. En esta internación presentaba dolor abdominal difuso más prominente en fosa ilíaca derecha con descompresión dolorosa, sometido a videolaparoscopia exploradora y evidenciaba apendicitis grado 4 . El análisis histopatológico mostró un tumor neuroendocrino en un apéndice G1 bien diferenciado con un tamaño de $2 \mathrm{~mm}$ en la eje mayor, índice mitótico con menos de 2 mitosis, estadificación patológica pT1 pNx, márgenes libres, invasión linfovascular y perineural. Inmunohistoquímica con índice de proliferación celular (Ki-67) del 1\% e identificación de los componentes Clon policlonal Cromogranina A y clon Sinaptofisina DARKSYNAP positivo. Conclusión: Presentamos el caso de un paciente con Tumor neuroendocrino de apéndice con presentación inicial como apendicitis aguda y, en patología, limitado al apéndice sin invasión linfovascular y sometido a apendicectomía total como procedimiento curativo.

Palabras clave: Apendectomía; Apendicitis; Células neuroendócrinas; Neoplasias; Proliferación celular.

\section{Introdução}

As neoplasias de apêndice, primárias e secundárias, são raras e são identificadas em cerca de $1 \%$ de todas as apendicectomias. Considerando os tumores primários, os tipos histológicos principais são: primeiro, os de linhagem epitelial e; segundo, os de linhagem neuroendócrina. A mais comum apresentação dessas neoplasias ocorre por apendicite aguda, evidenciada em $30-50 \%$ dos casos e ocorre, mais comumente, nos neuroendócrinos quando comparados aos epiteliais (Leonards et al, 2017).

Os tumores neuroendócrinos (TNE), também chamados de tumores carcinóides, são as neoplasias que acometem o apêndice com incidência anual de 2 a 5 por 100.000 pessoas, sendo encontrado em 0,2-0,7\% de todas as apendicectomias (Moris et al, 2018). São derivados de células de origem neuroendócrina, mais especificamente das células enterocromafins, apresentando secreções hormonais específicas a depender do seu sítio primário de origem. Tem curso indolente, por vezes, com sintomas inespecíficos, até que o tumor oclua o lúmen do apêndice e cerca de $50 \%$ dos pacientes confirmam o diagnóstico após apendicectomia por apendicite aguda (Abreu, 2018 e Goede et al, 2003). 
Estudos para manejo atuais demonstram que, $80 \%$ dos tumores apresentam diâmetro inferior a $1 \mathrm{~cm}$ ao diagnóstico, apresentando sobrevida em cinco anos favorável e requerem apenas apendicectomia simples para tratamento e, para aqueles com diâmetro superior a $1 \mathrm{~cm}$ necessitam, além da apendicectomia, acompanhamento pós-operatório por no mínimo 5 anos (Griniatsos, 2010).

O objetivo deste trabalho é relatar o caso de um paciente diagnosticado com tumor neuroendócrino de apêndice apos apendicite aguda perfurada que havia apresentado, anteriormente, tromboses de vasos venosos abdominais.

\section{Metodologia}

\section{Desenho do Estudo}

Trata-se de um estudo descritivo do tipo Relato de Caso, utilizando-se informações obtidas por meio de entrevista com o paciente durante a internação hospitalar, bem como dados obtidos no prontuário, os quais foram autorizados pelo paciente por meio de termo de consentimento livre e esclarecido assinado pelo paciente. Realizado no Hospital Geral Dr. Waldemar Alcântara (HGWA) em Fortaleza-CE em setembro de 2020.

\section{Relato de Caso}

Paciente de 28 anos, sexo masculino, há 2 meses com dor abdominal localizada em mesogástrio e epigástrio, diária e de intensidade moderada associada a distensão abdominal, procurou a emergência médica, onde foi prescrito analgesia e, em seguida, liberado para domicílio.

Contudo, houve persistência da dor e surgimento de uma febre não mensurada com calafrios cerca de uma semana depois. Por conta disso, procurou novamente a emergência médica, onde foi iniciado ciprofloxacino associado a metronidazol ambulatorialmente devido suspeita de infecção intra-abdominal.

Todavia, o paciente persistia com a dor, além da distensão abdominal. Assim, procurou mais uma vez o serviço de saúde, onde foram realizados por duas vezes, o exame de ultrassom abdominal total e, em ambas, não foram evidenciadas alterações. Diante disso, para continuidade da investigação, foi optado pela realização da tomografia de abdome com contraste que, por sua vez, demonstrou falha de enchimento no interior da veia portal principal, ramo esquerdo da veia porta e veia mesentérica superior, vesícula biliar, vias biliares pâncreas e apêndice cecal sem alterações. Sendo assim, iniciado anticoagulação com varfarina devido as tromboses intra-abdominais e referenciado para controle ambulatorial da dosagem de varfarina e para serviço de hematologia para investigação de trombofilias.

Após um mês, o paciente retorna ao ambulatório para ajuste de anticoagulante, relatando, na ocasião, há 2 dias distensão abdominal associada a dor abdominal difusa, mais proeminente em flanco direito com descompressão brusca dolorosa e decidido por nova internação hospitalar. Exames laboratoriais admissionais demonstravam hemoglobina $13.3 \mathrm{~g} / \mathrm{dl}$, hematócrito 38.9\%, leucócitos $14.500 / \mathrm{mm} 3$, plaquetas 203.000/mm3, proteína C reativa $8,4 \mathrm{mg} / \mathrm{dl}$, velocidade de hemossedimentação $60 \mathrm{~mm}$, ureia $23 \mathrm{mg} / \mathrm{dl}$, creatinina $0.72 \mathrm{mg} / \mathrm{dl}$. Requerido avaliação de urgência da cirurgia geral e iniciado ceftriaxona e metronidazol. Além disso, realizado ultrassonografia abdominal na urgência que não identificou apêndice cecal, no entanto, apresentava pequena quantidade de líquido livre na fossa ilíaca direita.

Dessa forma, foi realizado videolaparoscopia por suspeita de apendicite aguda em apêndice retrocecal, com achados de apêndice vermiforme edemaciado com perfuração e bloqueio com alças de delgado e pequena quantidade de líquido livre em goteira perietocólica direita, além de realização secção de apêndice, mesoapêndice e artéria apendicular, com envio de material para análise histopatológica. 
Paciente evoluiu em período de pós-operatório com melhora importante da distensão e dor abdominal, com boa progressão de dieta por via oral e sem complicações em sítio cirúrgico.

Relatório de patologia cirúrgica demonstra apendicite aguda supurativa com mesoapendicite (Figura 1) e Tumor neuroendócrino bem diferenciado G1 em metade distal do apêndice (Figura 2) com tamanho de $2 \mathrm{~mm}$ em seu maior eixo, índice mitótico com menos de 2 mitoses por campo com invasão de submucosa, estadiamento patológico pT1 pNx, margens cirúrgicas livres, invasão linfovascular e perineural ausentes.

Perfil imuno-histoquímico com índice de proliferação celular (Ki-67) de 1\% (Figura 3) e identificação dos componentes Cromogranina A clone policlonal e Sinaptofisina (Figura 4) clone DARKSYNAP positivos.

Figura 1. Imagem microscópica em aumento de 40x em coloração Hematoxilina e Eosina, exibindo parede de apêndice necrótica e com infiltrado inflamatório transmural, representando apendicite aguda.

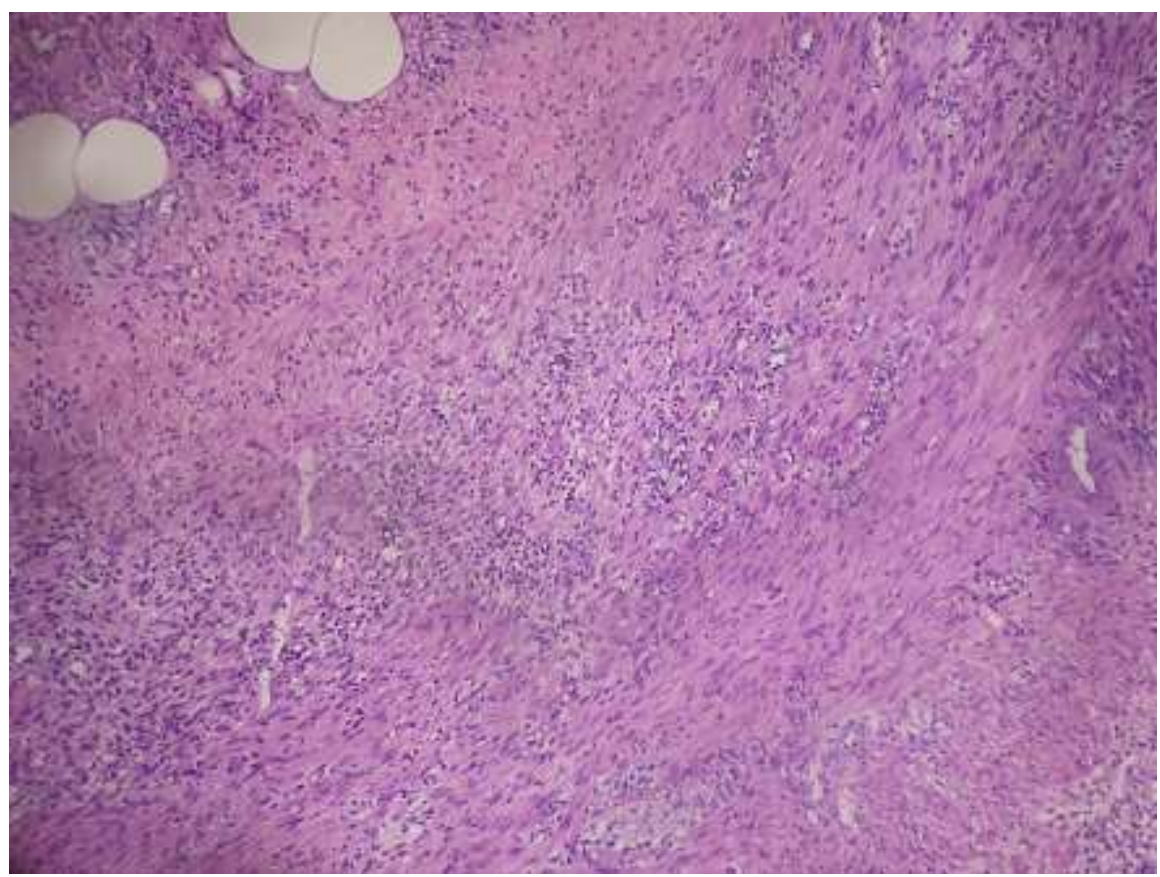

Fonte: Autores. 
Figura 2. Imagens microscópicas em coloração de Hematoxilina e Eosina. À esquerda aumento 40x, exibindo ilhotas de células epitelioides de aspecto neuroendócrino em parede muscular de apêndice cecal. À direita aumento 200x detalhando núcleo de células epitelioides com cromatina granular fino, sugerindo natureza neuroendócrina lesional.

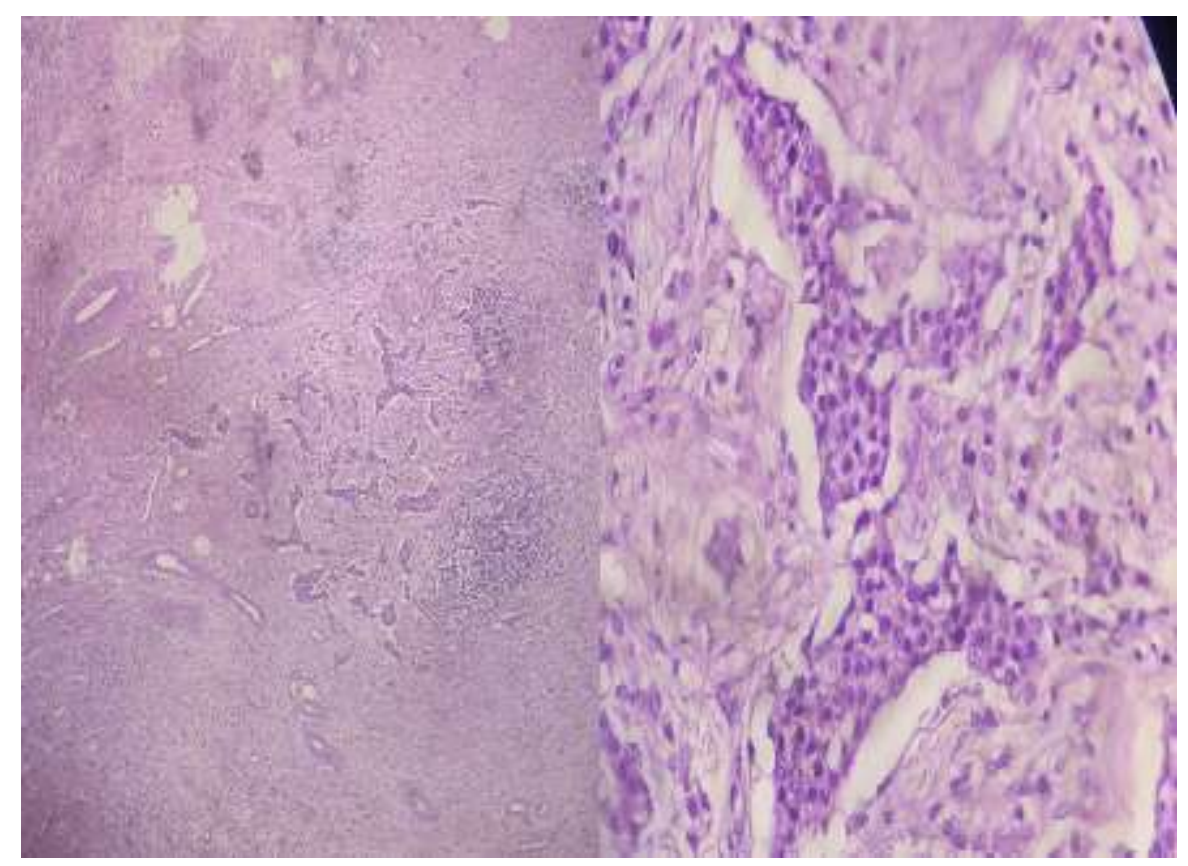

Fonte: Autores.

Figura 3. Imagem microscópica em aumento de 200x de reação imunohistoquímica com marcador Ki67, relevando baixo índice proliferativo de 2\%, confirmando diagnóstico de Tumor Neuroendócrino do apêndice de baixo grau.

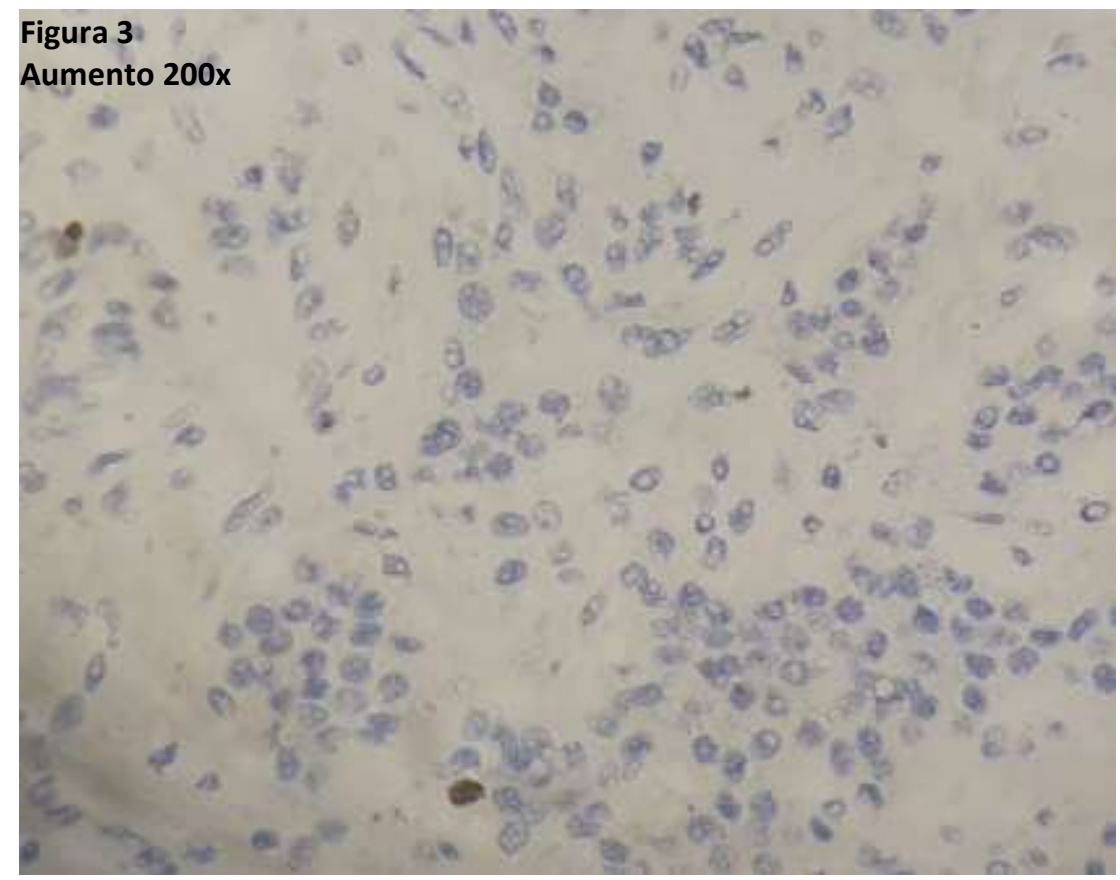

Fonte: Autores. 
Figura 4. Imagens microscópicas confirmando imunoexpressão neuroendócrina da células epitelioides em parede cecal. À esquerda aumento de 200x reação imunohistoquímica do marcador Cromogranina. À direita aumento de 200x reação imunohistoquímica do marcador Sinaptofisina.

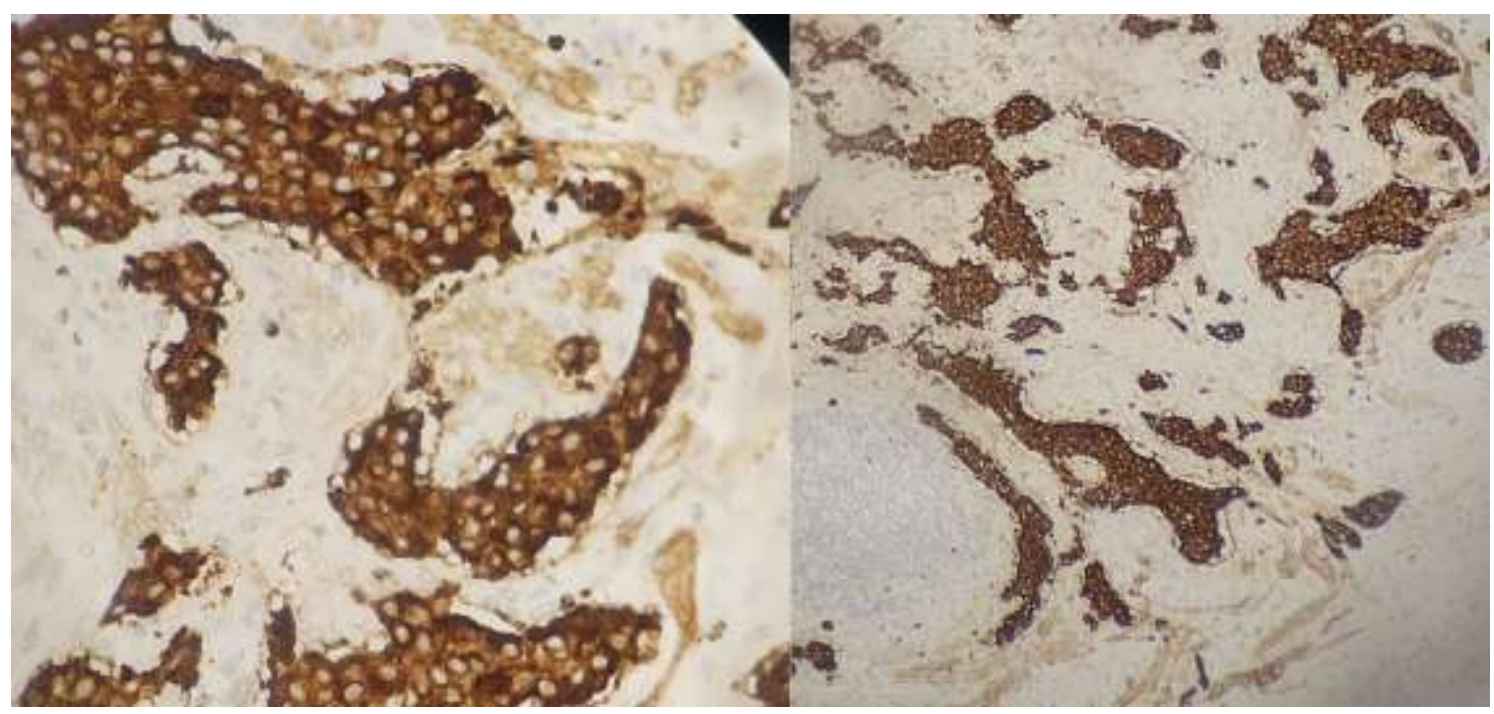

Fonte: Autores.

\section{Discussão}

As neoplasias de células neuroendócrinas de apêndice são raras e ocorrem, principalmente, em mulheres jovens na faixa etária de 15 a 19 anos, uma vez que são mais submetidas a laparoscopias que os homens, estes mais acometidos na faixa etária de 20 a 24 anos. Com incidência anual em torno de 2 a 3 casos por milhão de indivíduos são as neoplasias mais encontradas em biópsias do apêndice, representando entre 32-57\% dos tumores de apêndice (Stinner \& Rothmund, 2005).

Produzem grânulos com expressão de marcadores neuroendócrinos, como Cromogranina A e Sinaptofisina (Oronsky et al, 2017; Silva et al, 2010). A Cromogranina A é usada como marcador tumoral neuroendócrino e útil para diferenciar de outras neoplasias, principalmente nos quadros mais avançados (Modlin et al, 2006).

Não possuem uma etiologia definida, mas, apresentam influência genética com deleções do gene PLC $\beta 3$, causando crescimento descontrolado das células neuroendócrinas (Neto e Felippe, 2014). Classificados por graus de gravidade de acordo com sua atividade mitótica por campo e seu índice proliferativo (Ki-67), logo, representa o número de células em divisão dentro de um campo de 100 células (Di Domenico et al, 2014). Índice proliferativo de $2 \%$ ou inferior representa tumores bem diferenciados G1 de crescimento lento, índice entre 3\% e 20\% e considerado como moderadamente diferenciado G2 e são mais propensos a crescer e sofrer metástase e índice superior a 20\% são carcinomas neuroendócrinos pouco diferenciados G3 com células anormais com tendência de rápido crescimento e metástase (Foltyn et al, 2012).

A biópsia é o teste que define o diagnóstico da doença, enquanto o índice de proliferação celular através da quantificação de Ki67 auxilia no prognostico. Índices abaixo de $2 \%$ ocorrem em tumores de crescimento lento e acima de $10 \%$ sugerem crescimento acelerado (Pawa et al, 2018).

A maioria dos tumores sintomáticos é detectada devido à apendicite aguda. Apesar disso, a biópsia encontra um fator de obstrução em apenas $25 \%$ dos casos (O’Donnell et al, 2007). A síndrome carcinoide em associação com um tumor neuroendócrino apendicular bem diferenciado com metástase e extremamente rara, ocorrendo a referida síndrome em menos de $1 \%$ dos casos. No apêndice, a ponta do órgão é o local mais acometido. Tumores de grau 1 (marcador Ki-67 menor que $2 \%$ ) 
tem um prognostico excelente e geralmente podem ser removidos com segurança sem nenhuma intervenção cirúrgica adicional (Kloppel, 2011).

São o tipo de neoplasia com melhores desfechos comparados aos outros tipos de tumores que acometem o apêndice, principalmente devido a acometer indivíduos mais jovens, sexo feminino, doença geralmente limitada ao apêndice, detecção mais precoce e a biologia própria do tumor. O tamanho é considerado o melhor indicador para definir a extensão da cirurgia, o grau de disseminação é associado ao tamanho do tumor primário (Grozinsky-Glasberg et al, 2013).

Em relação a sobrevida em 5 anos, paciente com tumores neuroendócrinos apresentam taxa de $83.1 \%$ comparado a taxa de 49.2\% dos tumores não carcinoides (Alexandraki et al, 2016).

Quando estão limitados ao apêndice e com diâmetro inferior a $1 \mathrm{~cm}$ apresentam risco de metástase de praticamente zero. Em um estudo avaliando 31 pacientes não houve recorrência de doença regional ou metástase em um período de acompanhamento de 5 anos, sendo efetivamente tratados apenas com apendicectomia simples com sobrevida de $100 \%$ em 5 anos (Murray et al, 2014). Em outro estudo avaliando 150 pacientes, nenhum paciente com tumor de diâmetro inferior a $2 \mathrm{~cm}$ apresentou acometimento linfonodal ou metástases durante 25 anos de acompanhamento (Pawa et al, 2018).

Os pacientes acometidos com neoplasias entre $1 \mathrm{~cm}$ e $2 \mathrm{~cm}$ que realizaram apendicectomia e hemicolectomia direita sem envolvimento linfonodal nenhum acompanhamento se faz necessário. Quando apresenta acometimento linfonodal ou acometimento à distância se faz necessário acompanhamento a longo prazo. Também é recomendado acompanhamento para aqueles que não realizaram a hemicolectomia direita e apresentam fatores de risco: invasão vascular, localização em base de apêndice, invasão de mesoapêndice superior a $3 \mathrm{~mm}$ e tumor G2 (Pape et al, 2016).

Para os tumores com tamanho superior a $2 \mathrm{~cm}$ é recomendado realizar tomografia de abdome e cintilografia com análogos de somatostatina no sexto e décimo segundo meses do diagnóstico e, após isso, anualmente (Griniatsos e Michail, 2010). A colonoscopia de rotina para avaliar essas neoplasias não é indicada, sendo somente usada nos casos incomuns que cursam com invasão cecal e nos pacientes com indicação habitual de rastreio de câncer colorretal (Pape, Perren, Niederle, 2012).

\section{Conclusão}

Nesse relato de experiência clínico, o paciente se apresentava com apendicite aguda, a principal manifestação evidenciada nestas neoplasias, submetida a apendicectomia e com achado histopatológico de NET de $2 \mathrm{~mm}$ em seu maior diâmetro sem invasão linfovascular e perineural. Dessa forma, pelo conhecimento atual, nenhum tratamento adicional se faz necessário, tendo a apendicectomia como procedimento curativo com sobrevida de $100 \%$ nos próximos 5 anos e sem risco de recorrência para os próximos 25 anos.

\section{Referências}

Abreu, R. (2018). “Appendiceal neuroendocrine tumors: approach and treatment”. Journal of Coloproctology 38.4: $337-342$.

Alexandraki, K., Kaltsas, G., Grozinsky-Glasberg, S., Chatzellis, E., \& Grossman, A. (2016). Appendiceal neuroendocrine neoplasms: diagnosis and management, Endocrine-Related Cancer, 23(1), R27-R41.

Di Domenico, A., Wiedmer, T., Marinoni, I., \& Perren, A. (2017). Genetic and epigenetic drivers of neuroendocrine tumours (NET). Endocrine-Related Cancer 24, 9, R315-R334.

Foltyn, W., Zajęcki, W., Marek, B., Kajdaniuk, D., Siemińska, L., Zemczak, A., \& Kos-Kudła, B. (2012). The value of the Ki-67 proliferation marker as a prognostic factor in gastroenteropancreatic neuroendocrine tumours. Endokrynol Pol, 63(5):362-6. 
Goede, A. C., Caplin, M. E., \& Winslet, M. C. (2003). Carcinoid tumour of the appendix. Br J Surg, 90(11):1317-22.

Griniatsos, J., \& Michail, O. (2010) Appendiceal neuroendocrine tumors: Recent insights and clinical implications. World J Gastrointest Oncol, 2(4):192-6.

Grozinsky-Glasberg, S., Alexandraki, K. I., Barak, D., Doviner, V., Reissman, P., Kaltsas, G. A., \& Gross, D. J. (2013). Current size criteria for the management of neuroendocrine tumors of the appendix: are they valid? Clinical experience and review of the literature. Neuroendocrinology, 98(1):31-7.

Kloppel, G. (2011). Classification and pathology of gastroenteropancreatic neuroendocrine neoplasms. Endocr Relat Cancer, 18 Suppl 1:S1-16.

Leonards, L.M., Pahwa, A., Patel, M. K., Petersen, J., Nguyen, M. J., \& Jude, C. M. (2017). Neoplasms of the Appendix: Pictorial Review with Clinical and Pathologic Correlation. Radiographics, 37(4):1059-1083.

Modlin, I. M., Kidd, M., Latich, I., Zikusoka, M. N., Eick, G. N., Mane, S. M., \& Camp R. L. (2006). Genetic differentiation of appendiceal tumor malignancy: a guide for the perplexed. Ann Surg, 244(1):52-60.

Moris, D., Tsilimigras, D. I., Vagios, S., Ntanasis-Stathopoulos, I., Karachaliou, G. S., Papalampros, A., Alexandrou, A., Blazer, D. G., \& Felekouras, E., 3rd. (2018). Neuroendocrine Neoplasms of the Appendix: A Review of the Literature. Anticancer Res, 38(2):601-611.

Murray, S. E., Lloyd, R. V., Sippel, R. S., Chen, H., \& Oltmann, S. C. (2014). Postoperative surveillance of small appendiceal carcinoid tumors. Am J Surg, 207(3):342-345.

Neto, C., Felippe, I. S., et al. (2014). Carcinoid tumor of cecal appendix: one-year incidence at the Santa Marcelina Hospital.J. Coloproctol. (Rio J.) 34(4), 245-249. <http://www.scielo.br/scielo.php?script=sci_arttext\&pid=S2237-93632014000400245\&lng=en\&nrm=iso>.

Oronsky, B., Ma, P. C., Morgensztern, D., \& Carter, C. A. (2017). Nothing But NET: A Review of Neuroendocrine Tumors and Carcinomas. Neoplasia (New York, N.Y.), 19(12), 991-1002.

O'Donnell, M. E., Badger, S. A., Beattie, G. C., Carson, J., \& Garstin, W. I. (2007). Malignant neoplasms of the appendix. Int J Colorectal Dis, 22(10):123948.

Pawa, N., Clift, A. K., Osmani, H., Drymousis, P., Cichocki, A., Flora, R., Goldin, R., Patsouras, D., Baird, A. Malczewska, A., Kinross, J., Faiz, O., Antoniou, A., Wasan, H., Kaltsas, G. A., Darzi, A., Cwikla, J. B., \& Frilling, A. (2018). Surgical Management of Patients with Neuroendocrine Neoplasms of the Appendix: Appendectomy or More. Neuroendocrinology, 106(3):242-251.

Pape, U. F., Perren, A., Niederle, B., et al. (2012). ENETS consensus guidelines for the management of patients with neuroendocrine neoplasms from the jejuno-ileum and the appendix including goblet cell carcinomas. Neuroendocrinology, 95:135156.

Pape, U.F., Niederle, B., Costa, F., Gross, D., Kelestimur, F., Kianmanesh, R., Knigge, U., Öberg, K., Pavel, M., Perren, A., Toumpanakis, C., O'Connor, J., Krenning, E., Reed, N., O'Toole, D. Vienna Consensus Conference participants. (2016). ENETS Consensus Guidelines for Neuroendocrine Neoplasms of the Appendix (Excluding Goblet Cell Carcinomas). Neuroendocrinology, 103(2):144-52.

Silva, R. L., Linhares, E., Gonçalves, R., \& Ramos, C. (2010). Tumores Neuroendócrinos do Apêndice Cecal: Experiência do Instituto Nacional de Câncer. Revista Brasileira de Cancerologia, 56(4): 463-470.

Stinner, B., \& Rothmund, M. (2005). Neuroendocrine tumours (carcinoids) of the appendix. Best Pract Res Clin Gastroenterol, 19(5):729-38 